\title{
Assessing Donor Site Complications of Iliac Crest Bone Graft in Treatment of Scaphoid Nonunion
}

\author{
Davod Jafari, ${ }^{1}$ Hassan Ghandhari, ${ }^{1}$ Mahdi Abbaszadeh, ${ }^{1}$ Samad Joudi, ${ }^{1}$ Payam Hassany Shariat Panahy, ${ }^{2}$ \\ and Mohammad Reza Bahaeddini, ${ }^{1, *}$ \\ ${ }^{1}$ Bone and Joint Reconstruction Research Center, Shafa Orthopedic Hospital, Iran University of Medical Sciences, Tehran, IR Iran \\ ${ }^{2}$ University of Putra Malaysia, Serdang, Selangor, Malaysia \\ "Corresponding author: Mohammad Reza Bahaeddini, Bone and Joint Reconstruction Research Center, Shafa Orthopedic Hospital, Iran University of Medical Sciences, Tehran, \\ IR Iran. Tel: +98-9177103203, E-mail: mrbahaeddini@yahoo.com
}

Received 2016 April 18; Revised 2016 May 11; Accepted 2016 July 08.

\begin{abstract}
Background: Bone grafting is a common surgical technique to augment bone regeneration in orthopedic surgery. Autologous bone graft harvesting is the reliable treatment option and iliac crest is the most common harvesting site for healing bone fractures. However, the results of iliac crest bone graft harvesting are associated with morbidity and a number of complications.

Objectives: The aim of this study was to assess donor site complications of scaphoid nonunion, to find clinical outcomes and the impact of iliac crest bone graft on age, gender, pain, numbness, itching, nerve injury and scar appearance of patients.

Methods: In a prospective review of 61 cases of iliac crest bone graft procedures at the Shafa hospital from 2013 to 2014 , complications including pain, infection, hematoma, stress fracture, hypertrophic scars, numbness area and itching were assessed clinically. Results: Pain identified as the most common complication. The most common complications following postoperative pain at the donor site that reduced after six weeks were: numbness 3.27\%, unsatisfactory scar appearance $3.27 \%$ and itching discomfort $1.63 \%$. Conclusions: Harvesting of iliac crest bone graft can be the ideal way to prepare graft procedures required surgery scaphoid nonunion fractures with minimal complications. Nevertheless, with an adequate preoperative planning and appropriate surgical technique, the prevalence of these complications can be reduced.
\end{abstract}

Keywords: Donor Site Complications, Iliac Crest, Scaphoid Nonunion

\section{Background}

Each year 200000 bone-graft techniques are performed in the United States (1). The iliac crest bone graft is used as an adjunct treatment of scaphoid nonunion fracture (2). It remains the most common donor site for autogenous bone graft and provides sufficient quantities of cortical and cancellous bone for most clinical settings (3). However, despite strong reports of clinical success, it has limitations, including donor-site morbidity, nerve injury, pain, hematoma and infection (1).

The most significant point is that iliac crest is easily accessible. This means large amount of both cortical and cancellous bone can be harvested safely for reconstructing the fractures with only minimal donor-site morbidity $(3,4)$. Bone grafts help to promote bone healing in fractures and if transplanted in bone cells with sufficient available space, it can repair and regenerate itself (5). Bone grafts provide structural support for bone reconstruction and complete reconstruction is involved in bone conduction and induction.

Iliac crest bone harvesting during open surgery is a safe method with a rare complication. A few reports of this minor complication consist of gait disorders, stress fractures, blood loss, itching, hematoma, unsatisfactory appearance (deformity), and acute and chronic pain in the area $(6,7)$. Moreover, the iliac crest bone graft harvest is associated with significant complications, including perforation of the peritoneum, hernia and hip fractures $(8,9)$.

The effect of bone graft harvest on the patient depends on factors such as the amount of harvested grafts, surgical approach and procedure of cutting anterior and posterior edge, and performing a precise and comprehensive incision surgery $(3,10)$.

\section{Objectives}

In this study, we aimed to assess donor site complications of scaphoid nonunion, to find clinical outcomes and the impact of iliac crest bone graft on age, gender, pain, numbness, itching, nerve injury and scar appearance of patients. 


\section{Methods}

This was a prospective study with institutional review board number 1605. The sixty-one patients, who participated in this study, were selected randomly followed up for 12 weeks at the department of orthopedics from March 2013 to November 2014.

Patients were informed about their participation rights and informed consent was obtained from all candidates. According to the recommendations of the statistician, the minimum sample size was determined. The prospective cohort study on 61 patients with iliac crest bone grafting surgery complications was analyzed. The questionnaire consisted of two sections: socio demographic information including age, gender, type of surgery and approach, donor sites, and surgical complications such as pain. The major considered complications were infection, hematoma, stress fracture, hypertrophic scars, numbness area and itching, and complications associated with daily activities (disorder walking, doing housework, leisure activities and sexual activity). Furthermore, rare complications were assessed during the operation and on the third, second, sixth and twelfth week after the operation and finally on the last visit.

The amount and intensity of pain were determined by using the visual analog scale (VAS).

The procedure was performed with osteotomy of the center of iliac crest within $5 \mathrm{~cm}$ from superior anterior iliac spine to curettage and harvest cancellous bone. Inner and outer tabulation were maintained intact during graft harvesting.

The results were analyzed using the SPSS version 16 statistical software. Student t test with confidence level of 95\% was used to assess the preoperative and postoperative quantitative data. In addition, the qualitative data was assessed using the chi-square test. The significant probability was considered as less than $0.5 \%$.

\section{Results}

The average age of the study population was 58 years (range: 16, 60). Fifty-eight patients (95\%) were male with a mean age of 27.93 and three were female (5\%) with mean age of 28.33 years. In 31 patients (50.8\%), a bone graft had been taken from the right iliac bone, and in 30 patients (49.2\%) from the left iliac bone.

Pain was the most common complication of this study. However, the pain intensity and the number of patients who had pain decreased over time. Six weeks after the operation, seven patients (11.4\%) had a chronic pain and 54 (88.9\%) had no pain. Chronic pain for more than three months was observed in one patient (1.6\%), as it is associated with less pain intensity. The average pain intensity in women was higher than men at clinical follow-up. Results from the cross correlation analysis of mean of pain in males and females during the follow up time are shown in Table 1.

Based on cross correlation analysis, there was a linear correlation between pain intensity and female gender till the sixth week, because after the sixth week, there was no female to follow up; there are no analysis and reports about this correlation after the sixth weeks. The results of cross coefficient between pain and gender are shown in Figure 1.

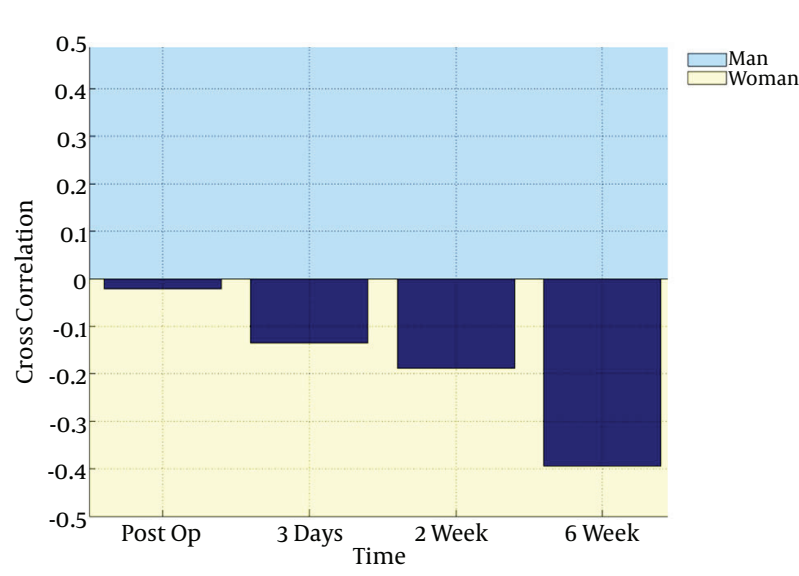

Figure 1. Results of Cross Coefficient Between Pain and Gender

In addition, as depicted in Figure 2, there was a linear correlation between pain intensity and age until the sixth week and the pain intensity also increased with increasing age. Because the number of patients decreased dramatically after the sixth week; there is no analysis and report about this correlation.

The following evaluation showed two patients (27/3\%) had numbness and one patient (1.63\%) had itching discomfort. Furthermore, unsatisfactory scar appearance was observed in two patients (3.27\%).

\section{Discussion}

Iliac crest bone grafts are well recognized as the gold standard to regenerate bone in orthopedic surgery (6). Since, harvesting of iliac crest bone graft is associated with higher success rates of bone graft, the complications of grafting have been accepted largely as a technique in orthopedic operation. Iliac crest bone grafts have become increasingly important because the results of bone graft approaches have a high rate of success; low risks and associated costs (6). This study was designed to assess donor site 
Table 1. Mean Pain in Males and Females During the Follow up Period

\begin{tabular}{lccccc}
\hline & & \multicolumn{3}{c}{ Cross Correlation } \\
\cline { 2 - 6 } Gender & Post Op & 3 Days & 2 Weeks & 6 Weeks & 12 Weeks \\
\hline Male & 6.79 & 3.43 & 1.08 & 0.08 & 0.03 \\
Female & 7 & 4.33 & 2 & 0.66 & - \\
Total & 6.80 & 3.47 & 1.13 & 0.11 & 0.01 \\
\hline
\end{tabular}

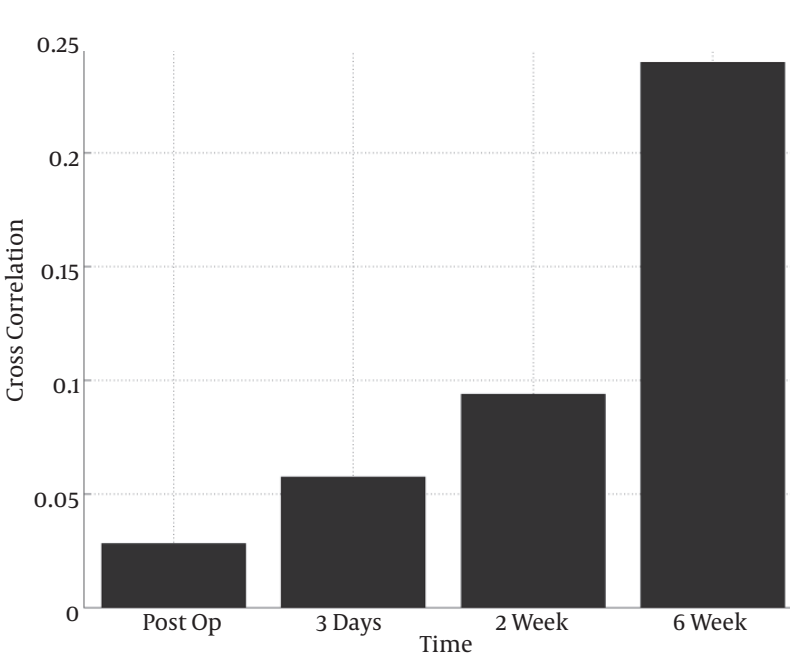

Figure 2. Results of the Cross Coefficient Between Pain and Age

complications in 61 patients in the treatment of scaphoid nonunion.

Pain was the most frequently cited complication of harvesting iliac crest bone grafts (6). While, Palmer et al. reported $6.16 \%$, Schwartz et al. reported 19\%, and Schaaf et al. reported only $4 \%$ of their patients having had chronic pain, our study showed the lowest level of donor site pain (11-13). We believe that this result was affected by many factors as these factors impact on people's pain threshold. Among these factors, genetics and race impact people's pain threshold and the amount of harvested graft influences the intensity of pain (13). In addition, gender also effects pain tolerance and based on our results there was a linear relationship between female gender and pain intensity. This finding is also consistent with the study of Schaaf et al. (12).

Furthermore, assessing numbness as another complication of iliac crest bone grafts showed that $3.27 \%$ of patients had temporary numbness. This result is consistent with the study of Schaaf, who reported $2.7 \%$ of their patients had numbness (12). However, as Schwartz reported that numbness exists in $24 \%$ of their patients (13), we be- lieve that the low rate of numbness in our study is related to the low amount of harvested grafts and more likely related to performing precise and comprehensive incision surgery.

Assessing scar appearance showed that $3.27 \%$ of patients had unsatisfactory scars and this result is consistent with Palmer and Schwartz reports (3\% and 5\%, respectively) $(11,13)$.

Based on our study, $1.63 \%$ of patients had itching, which is similar to the rate reported by Palmer (3.3\%) (11).

In general, a lower rate of complications was observed in this study. This is because the amount of the harvested graft was low and we made a shallow incision to harvest the graft. Therefore, the amount of graft and the type of incision significantly reduced the intensity of pain, nerve injury and unsatisfactory scar appearance.

This study had several limitations, mostly stemming from the effects of major factors that influence on evaluation results such as cultural differences, expectations of the appearance and physical structure. However, we think that there is no uniform gold standard in postoperative pain management. Therefore, further research needs to be conducted to compare and illustrate more accurate results.

Therefore, iliac crest bone grafting is considered a more useful technique to treat the scaphoid nonunion fractures.

The evaluation of donor site complications of iliac crest bone grafting has shown that gender is a factor affecting pain, and women have more pain than men. Proceeding from these results, it should be concluded that iliac crest bone grafting is the ideal way to treat scaphoid nonunion fractures with minimal complications.

\section{Acknowledgments}

The authors would like to express their special thanks to all patients who took part in this study and the medical personnel of the orthopedic surgery ward of Shafa orthopedic hospital for their cooperation and providing facilities to perform this study. 


\section{Footnote}

Authors' Contribution: Study concept and design, Davod Jafari, Hassan Ghandhari and Mahdi Abbaszadeh; analysis and interpretation of data, Samad Joudi, Payam Hassany Shariat Panahy and Mohammad Reza Bahaeddini; drafting of the manuscript, Samad Joudi and Payam Hassani Shariat Panahy; critical revision of the manuscript for important intellectual content and study supervision, Hassan Ghandhari.

\section{References}

1. Myeroff C, Archdeacon M. Autogenous bone graft: donor sites and techniques. J Bone Joint Surg Am. 2011;93(23):2227-36. doi: 10.2106/JBJS.J.01513. [PubMed: 22159859].

2. Lementowski PW, Lucas P, Taddonio RF. Acute and chronic complications of intracortical iliac crest bone grafting versus the traditional corticocancellous technique for spinal fusion surgery. Orthopedics. 2010;33(4).

3. Ahlmann E, Patzakis M, Roidis N, Shepherd L, Holtom P. Comparison of anterior and posterior iliac crest bone grafts in terms of harvest-site morbidity and functional outcomes. J Bone Joint Surg Am. 2002;84(5):716-20.

4. Finkemeier CG. Bone-grafting and bone-graft substitutes. J Bone Joint Surg Am. 2002;84-A(3):454-64. [PubMed: 11886919].

5. Klokkevold PR, Jovanovic SA. Advanced implant surgery and bone grafting techniques. 9 ed. Saunders; 2002.
6. Goulet JA, Senunas LE, DeSilva GL, Greenfield ML. Autogenous iliac crest bone graft. Complications and functional assessment. Clin Orthop Relat Res. 1997(339):76-81. [PubMed: 9186204].

7. Nkenke E, Weisbach V, Winckler E, Kessler P, Schultze-Mosgau S, Wiltfang J, et al. Morbidity of harvesting of bone grafts from the iliac crest for preprosthetic augmentation procedures: a prospective study. Int J Oral Maxillofac Surg. 2004;33(2):157-63. doi: 10.1054/ijom.2003.0465. [PubMed: 15050072].

8. Porchet F, Jaques B. Unusual complications at iliac crest bone graft donor site: experience with two cases. Neurosurgery. 1996;39(4):8569. [PubMed: 8880782].

9. Burstein FD, Simms C, Cohen SR, Work F, Paschal M. Iliac crest bone graft harvesting techniques: a comparison. Plast Reconstr Surg. 2000;105(1):34-9. [PubMed:10626967].

10. Kessler P, Thorwarth M, Bloch-Birkholz A, Nkenke E, Neukam FW. Harvesting of bone from the iliac crest-comparison of the anterior and posterior sites. Br J Oral Maxillofac Surg. 2005;43(1):51-6. doi: 10.1016/j.bjoms.2004.08.026. [PubMed: 15620775].

11. Palmer W, Crawford-Sykes A, Rose RE. Donor site morbidity following iliac crest bone graft. West Indian Med J. 2008;57(5):490-2. [PubMed: 19565981].

12. Schaaf H, Lendeckel S, Howaldt HP, Streckbein P. Donor site morbidity after bone harvesting from the anterior iliac crest. Oral Surg Oral Med Oral Pathol Oral Radiol Endod. 2010;109(1):52-8. doi: 10.1016/j.tripleo.2009.08.023. [PubMed: 20123379].

13. Schwartz CE, Martha JF, Kowalski P, Wang DA, Bode R, Li L, et al. Prospective evaluation of chronic pain associated with posterior autologous iliac crest bone graft harvest and its effect on postoperative outcome. Health Qual Life Outcomes. 2009;7:49. doi: 10.1186/1477-75257-49. [PubMed: 19480692]. 